\title{
Self-organized criticality judgment and extreme statistics analysis of major urban fires
}

\author{
WANG JingHong ${ }^{1,2}$, XIE Shu $^{3} \&$ SUN JinHua ${ }^{1 *}$ \\ ${ }^{1}$ State Key Laboratory of Fire Science, University of Science and Technology of China, Hefei 230026, China; \\ ${ }^{2}$ USTC-CityU Joint Advanced Research Centre, Suzhou 215123, China; \\ ${ }^{3}$ Anhui General Fire Brigade, Hefei 230026, China
}

Received January 13, 2010; accepted April 1, 2010

\begin{abstract}
The spatial (economic loss) and temporal characteristics of urban fires were analyzed employing relevant statistical methods. A fractal structure in terms of the power-law relation between fire frequency and economic loss was found on a spatial scale, and an exponential relation between frequency and time interval was found on a temporal scale. Thus, urban fire does not meet the rigorous criteria of self-organized criticality. In addition, based on the spatial power-law distribution characteristics, a correlation model of the frequency and scale of loss due to urban fire was established using the extremum statistical method. This model was then applied to the case analysis of Hefei and the probability of major fire incidents in the future was predicted.
\end{abstract}

self-organized criticality, extreme statistics, correlation model, major urban fires

Citation: Wang J H, Xie S, Sun J H. Self-organized criticality judgment and extreme statistics analysis of major urban fires. Chinese Sci Bull, 2011, 56: 567-572, doi: $10.1007 / \mathrm{s} 11434-010-4062-\mathrm{y}$

Self-organized criticality (SOC), a concept originally introduced by Bak et al. [1] in 1987, is a general theory to describe the ubiquity of fractals [2] and $1 / f$ noise as natural coupled degrees of freedom. Since the concept of SOC was proposed, many scholars in different fields have conducted a wide range of research on actual systems and phenomena that exhibit the characteristics of SOC. Examples include the failure model of complex electric power systems [3], the model of the spread of an epidemic [4], the sandpile model [5], and the forest fire model [6,7]. Common features of these models are that the systems contain a number of elements that play short-term roles, and that there is spontaneous evolution toward a critical state in which a series of disturbances (i.e. slow driving or energy input) causes "avalanche" events. When a system reaches equilibrium, these "avalanche-like" dissipation events demonstrate fractal structure, or specifically, a power-law distribution on the spatial scale and simultaneously $1 / f$ noise on the temporal

*Corresponding author (email: sunjh@mail.ustc.edu.cn) scale. Research on urban fire systems has shown that the frequency-size distribution of urban fires satisfies a power-law relation [8], whereas there has been no specific investigation into whether the urban fire system has power-law distributions on both spatial and temporal scales; that is, whether the urban fire system meets the rigorous criteria of SOC.

The statistics of extremes are mainly used to obtain the maximum and minimum values of a sample of a certain size, where the sample may be simulated through stochastic variables with respective probability distributions. For some stochastic events that rarely happen but have a tremendous impact when they do, the extreme value analysis is an effective approach for modeling their extreme variability and carrying out statistical analysis. Such analysis can thereby provide a basis of theoretical assessment to system managers. The statistics of extremes have been widely used in investigations of earthquakes, floods, and other natural disasters, as well as the fields of insurance and finance [9-12]. However, extreme value theory has not yet been completely 
introduced to the analysis of urban fire incidents. The maximum value for urban fire is of importance because the focus is usually on major fires, and in particular, their probability of occurrence. Previous studies have attempted to predict the frequency of and damage done by urban fires using artificial neural networks, grey theory, Markov chains, information diffusion theory, and other methods [13-15]. However, those research efforts focused on the overall trend of urban fires without taking into account the differences and relationships between small fires and major fires. In this paper, we analyze the spatial and temporal distributions of characteristics of an urban fire system, on the basis of which, the theory of extreme value statistics is applied to the urban fire prediction. In particular, we make the first attempt to predict major fires from information on small fires with a view to provide suggestions for urban fire prevention.

\section{SOC of an urban fire system}

\subsection{Fractal structure of an urban fire system on a spa- tial scale}

All cities can be divided into many regions with different functions and characteristics. Thus, the analysis of fire data for an entire city sometimes does not reflect the fire features of a certain urban area. This is because there is an integrated offset for a number of factors when using a large sample population for statistical analysis. Therefore, this paper analyzes not only fire data for a whole city but also a sample representative of certain regional characteristics in the city so that the results can better reflect the differences and relationships between using large and small sample populations of fire data. The original fire data analyzed in this paper were obtained from the Anhui General Fire Brigade. The data range from 2000 to 2008 and include information on the time of fire ignition, location of fire ignition, cause of fire, and direct economic impact. As the data from 2003 to 2006 are not sufficiently integrated owing to the continual updating of the fire recording system, only the data of 2000 , 2001, 2002, 2007 and 2008 were analyzed in this paper.

As the small sample population, fire data for the Shushan

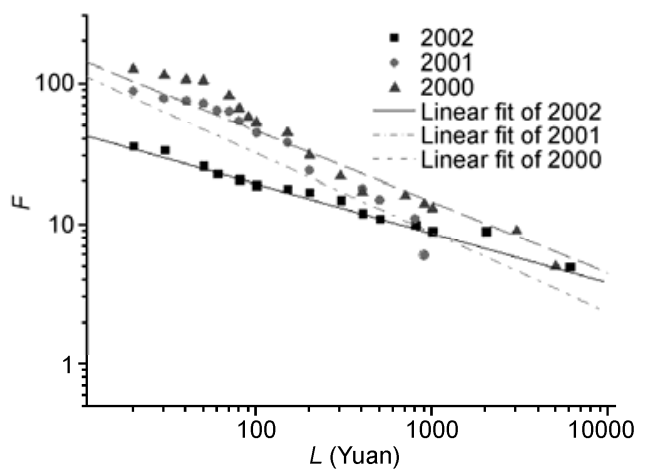

region in Hefei are analyzed and the frequency-economic loss distribution (hereafter simply referred to as the frequency-loss distribution) on an appropriate scale is given. It is obvious that this distribution satisfies a power-law relation in double logarithmic coordinates, as shown in Figure 1. The economic loss due to a fire is a reflection of the scale of the fire, or in other words, the spatial characteristics of the fire. Let $L$ be the economic loss and $F$ be the frequency of fire incidents with the loss exceeding $L$. The power-law relation between $F$ and $L$ is then

$$
F \propto L^{-\alpha} .
$$

Results of the linear regression of data points obtained employing the ordinary least squares (OLS) method are shown as straight lines in Figure 1. The line of best fit for fire data in 2000 is a straight line with a slope of $-\alpha_{1}=$ $-0.50662 \pm 0.03836$ (i.e. $\lg F \propto-0.5066 \times \lg L$ ), with the square of the correlation coefficient exceeding 0.9. In addition, the fitting results for fire data in 2001, 2002, 2007 and 2008 are $-\alpha_{2}=-0.56644 \pm 0.06575,-\alpha_{3}=-0.35401 \pm 0.02145,-\alpha_{4}=-0.5665$ \pm 0.0357 , and $-\alpha_{5}=-47644 \pm 0.02683$, with the square of the correlation coefficient exceeding 0.9 . These results indicate that the power-law relation of the fire frequency-loss distribution is stable on a spatial scale because the numerical value of the power-law relation for the three consecutive years 2000 to 2002 and for the two consecutive years 2007 and 2008 is almost constant; the line of best fit has a gradient with a range of approximately -0.354 to -0.566 , an average value of -0.494 , and a standard deviation of 0.0874 . Based on such a stable power-law relation, the frequency of urban fires and the scale of economic loss due to the urban fires can be reasonably predicted.

Similarly, the large sample population of fire data for the whole city is also analyzed in terms of the fire frequencyloss distribution on a spatial scale. Figure 2 shows the results of data analysis of fire incidents with direct economic loss between thousands and millions of RMB. It is seen that the fire frequency-loss distribution for the whole city satisfies the same power-law relation satisfied by the distribution for the Shushan region. The gradients of lines of best fit are: $-0.48 \pm 0.00735$ (in 2008), $-0.52 \pm 0.00583$ (in 2007), $-0.42 \pm$

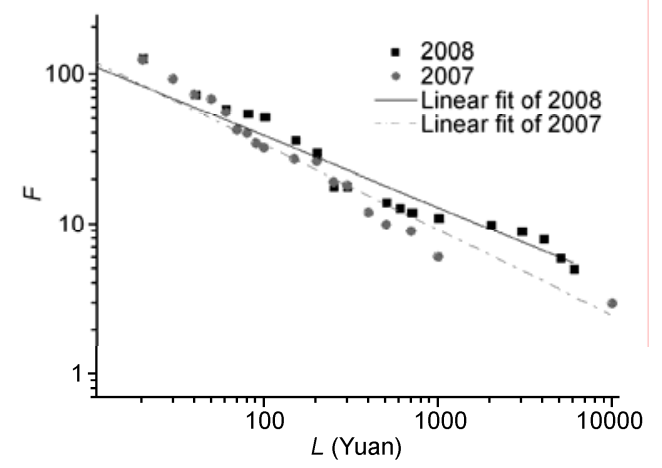

Figure 1 Annual fire frequency-loss distribution of the Shushan region in Hefei. 

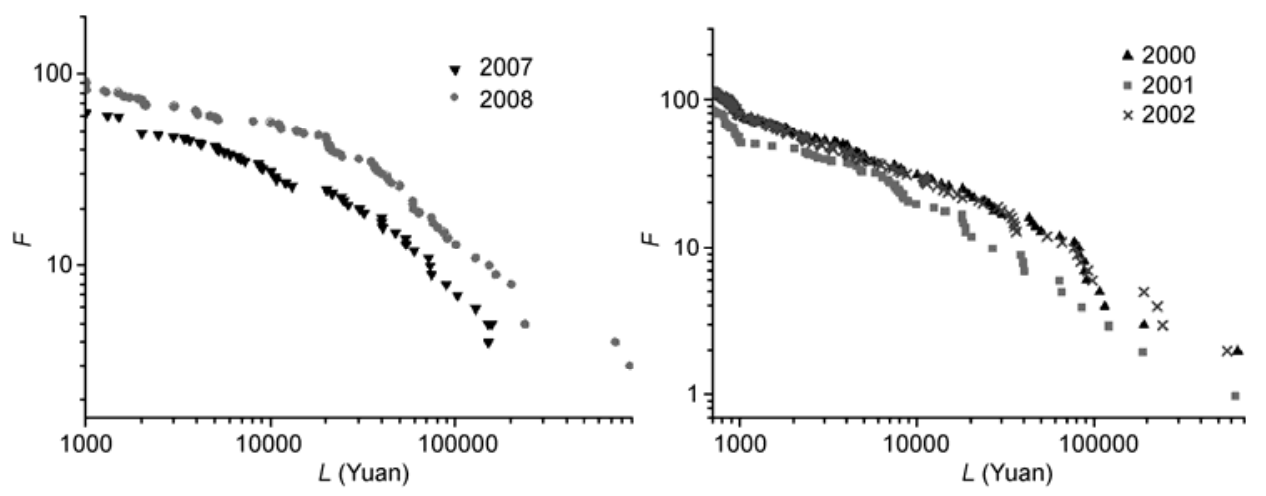

Figure 2 Annual fire frequency-loss distribution of the whole city of Hefei.

0.0071 (in 2002), $-0.475 \pm 0.0093$ (in 2001), and $-0.4 \pm$ 0.00602 (in 2000), which indicate that the spatial power-law distribution for the whole city is also stable with time.

It should be pointed out that the relatively stable power-law relation does not apply to the major fires in a city, particularly major fires with direct economic losses up to millions of yuan and sometimes even as high as 10 million yuan. Therefore, when investigating the spatial characteristics of an urban fire system, such major fires with low frequency are removed from power-law analysis; i.e. the impact of major fires on the fractal structure of the urban fire system is ignored. Instead, the statistics of extremes are applied to analyze the major fires.

By integrating all of the five-year fire incidents that caused direct economic loss of more than 1000 yuan to investigate the characteristics of urban fires in Hefei, it is found that the frequency-loss distribution of urban fires has a segmented power-law relation; i.e. the gradient of the line of best fit changes at some turning point with an increase in the scale of loss, as shown in Figure 3, which is consistent with previous results. Because major fires that cause direct economic loss of millions of yuan or even tens of millions of yuan are low-probability events in a medium-sized city

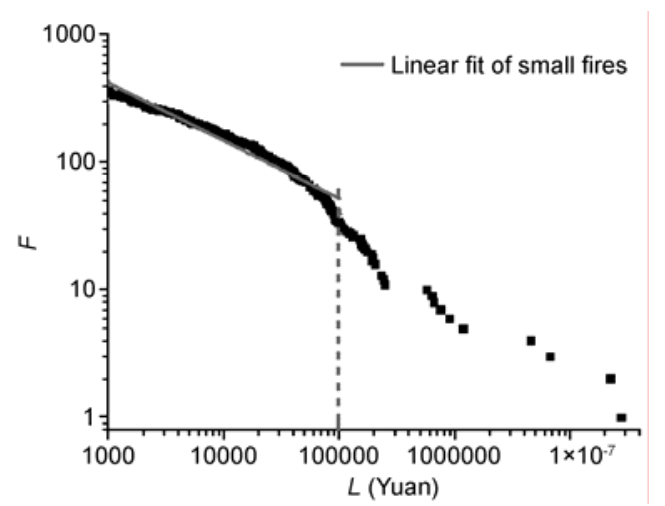

Figure 3 Five-year fire frequency-loss distribution for the whole city of Hefei. such as Hefei, the data points on the tail of the curve in Figure 3 are discrete.

\subsection{Fractal structure of an urban fire system on a temporal scale}

There are two criteria for SOC: a fractal structure of the system on a spatial scale (i.e. some power-law distribution) and $1 / f$ noise as the temporal counterpart of fractals. The $1 / f$ noise indicates that the power spectral density of a sequence is inversely proportional to the frequency. Although the sequence is disordered locally, there is negative correlation from a macroscopic view; i.e. the Fourier transform of the temporal correlation function is of the form $1 / f^{\alpha}$ where $\alpha \approx 1$. A series of urban fire incidents can be viewed as a chronologically ordered sequence of events, whose power spectral density can then be considered as aggregation levels of these events in various frequency bands (time periods).

We chronologically ordered the whole city's fire data for 2007 and 2008 and generated a sequence of time intervals $\left\{\tau_{i}\right\}$ (i.e. $\tau_{i}=t_{i+1}-t_{i}$, where $t_{i}$ is the time of the $i$ th fire incident. We define $F(\tau)$ as the frequency of occurrence of two fire incidents separated by time $\tau$, thus, $F(\tau)$ $=-D N\left(\tau^{\prime}>\tau\right) / D \tau$, where $N\left(\tau^{\prime}>\tau\right)$ represents two fire incidents whose time interval is greater than $\tau$. It is seen in Figure 4 that the frequency-time interval distribution of urban fire does not satisfy a power-law relation in double logarithmic coordinates, which indicates that the urban fire system does not have a temporal fractal pattern; i.e. no $1 / f$ noise exits. However, a linear relation is found between the logarithm of frequency and the time interval in logarithmic-linear coordinates, which indicates that the frequencytime interval distribution of urban fires satisfies an exponential relation that is also stable with time.

In conclusion, the urban fire system does not meet the rigorous criteria of SOC. The urban fire system has a spatial fractal structure but no temporal counterpart (i.e. $1 / f$ noise). However, there is a stable exponential distribution on the temporal scale instead of a power-law distribution. 


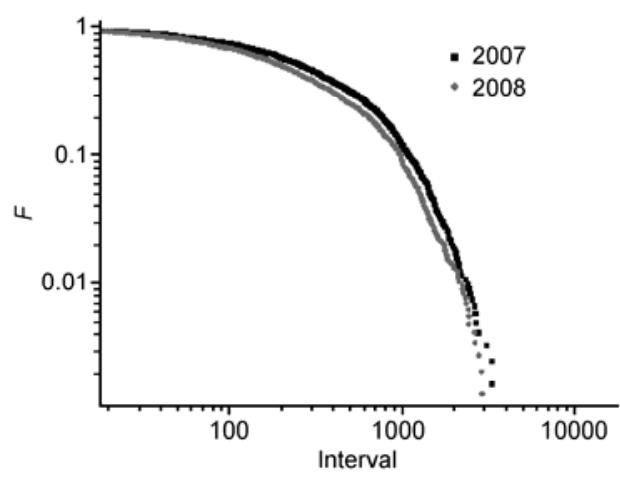

Figure 4 Fire frequency-time interval distribution for the whole city of Hefei.

\section{Extreme value theory and its distribution based on power-law behavior}

\subsection{Theory of the statistics of extremes}

The statistics of extremes is an area of statistical mathematics that deals with the behavior of the largest and/or smallest values of a given sample population. Our work is concerned with "large" extremes, namely the maximum economic loss due to a major fire in a city, and we therefore only discuss the statistics of extremes for largest values; i.e. we wish to find the probability distribution of the maximum value of a set of observations from a sample of $n$ independently and identically distributed (IID) random variables. Let $X_{1}, X_{2}, \cdots, X_{n}$ be a sequence of IID random variables with probability distribution function $F(x)$. We consider the distribution of the maximum of $\left\{X_{i}\right\}, Y_{n}=\max \left(X_{1}, X_{2}\right.$, $\left.\cdots, X_{n}\right)$, which is also a random variable. Thus, the distribution function of $Y_{n}$ is

$$
F(y)=P\left(Y_{n} \leqslant y\right)=P\left(X_{1} \leqslant y, X_{2} \leqslant y, \cdots, X_{n} \leqslant y\right) .
$$

When $0 \leqslant F(x)<1$ or $F(x)=1$, we have $F(y) \rightarrow 0$ or 1; i.e. $F(y)$ has a limited or asymptotic distribution. According to the Fisher-Tippett theorem of extremes, there are three types of extremal distributions of $F(y)$ referred to as Gumbel (I), Frechet (II), and Weibull (III). The criteria of each type of extremal distribution are:

Criterion I (convergent to type I) $\lim _{x \rightarrow \infty} \frac{\mathrm{d}}{\mathrm{d} x}\left[\frac{1}{h_{n}(x)}\right]=0$,

Criterion II (convergent to type II) $\lim _{x \rightarrow \infty} x h_{n}(x)=k$, where $k>0$ is a constant,

Criterion III (convergent to type III) $F_{X}(w)=1$ and $\lim _{x \rightarrow \infty}(x-w) h_{n}(x)=k$, where $w$ is the upper limit and $k>0$ is a constant.

$h_{n}(x)$ in the above three criteria is the disaster function of $X$, and considering the maximum we have

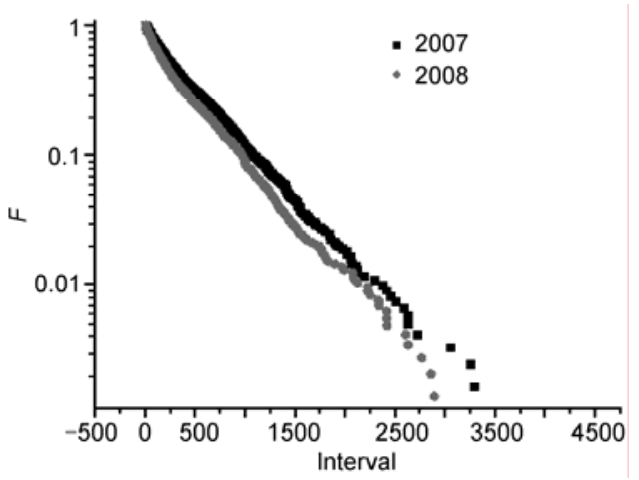

$$
h_{n}(x)=\frac{f_{X}(x)}{1-F_{X}(x)} \text {. }
$$

As a result, it is necessary to first identify the statistical distribution characteristics and functions of hazards before using the method of extremal statistics to find the distribution of the maximum loss value. Considering the known laws of urban fires (i.e. the power-law characteristics of the frequency-loss distribution), an accurate description of the trends of major urban fires is feasible.

\subsection{Distribution of urban fire extreme values based on the power-law behavior}

According to the power-law relation $F=C L^{-\alpha}$ (where $C$ is a constant) between the urban fire frequency $F$ and scale of loss $L$, we define $L_{\max }$ and $L_{\min }$ as the maximum and minimum losses among the data population. Let $X=\ln L$; then $F=C \mathrm{e}^{-\alpha X}$, where $X \geqslant X_{\min }$ and $X_{\min }=\ln L_{\min }$ (generally can be zero). Substituting the probability with the frequency, the distribution function of $X$ becomes

$$
F(x)=P(X \leqslant x)=\frac{\int_{X_{\min }}^{x} C \mathrm{e}^{-\alpha X} \mathrm{~d} X}{\int_{X_{\min }}^{\infty} C \mathrm{e}^{-\alpha X} \mathrm{~d} X}=1-\mathrm{e}^{-\alpha\left(x-X_{\min }\right)} .
$$

Here the numerator represents the cumulative number of occurrences with the logarithm of the scale of loss less than or equal to $x$ and the denominator represents the total number of events. Furthermore, the probability density function of $F(x)$ is

$$
f(x)=F^{\prime}(x)=\alpha \mathrm{e}^{-\alpha\left(x-X_{\min }\right)} .
$$

Accordingly, the disaster function $h_{n}(x)$ can be calculated and put into the criteria of the three types of extreme value distributions. It is found that criterion I is satisfied, indicating that the extreme value distribution of urban fires converges to the Gumbel formula, which can be further de- 
rived as

$$
F(y)=\exp (-\exp (-a(x-b)))
$$

where $a$ and $b$ are constants that can be calculated employing the following method.

We suppose a series of observational data corresponding to $n$ units of time and select the fire incident with the largest loss in every time unit. Thus, the sequence of largest losses $x_{j}(j=1,2, \cdots, n)$ where $x_{1} \leqslant x_{2} \leqslant \cdots \leqslant x_{j} \leqslant \cdots \leqslant x_{n}$ is obtained. It can be proved that $G\left(x_{j}\right)$, the mathematical expectation of the distribution function of $x_{j}$, is $\frac{j}{n+1}$, which yields

$$
-\ln \left(-\ln \frac{j}{n+1}\right)=a\left(x_{j}-b\right)
$$

Based on the above analysis, we choose one year as one unit time and put the largest loss of every year into eq. (7). Consequently we have $n$ linear equations for $a$ and $b$, which can be solved employing the OLS method. Therefore, another formula related to urban fire can be derived when the values of $a$ and $b$ are known; for example, the occurrence probability of fire incidents with the logarithm of direct economic loss not less than $M\left(M=\ln L_{\max }\right.$, where $L_{\max }$ is the loss) is

$$
P(M)=1-\exp (-T \exp (-a(M-b))) .
$$

\subsection{Case analysis}

From the preceding analysis on the relation between urban fire frequency and economic loss, we know that the urban fire system has relatively stable characteristics of the power-law distribution on a spatial scale and there is a turning point of this power-law relation near a direct economic loss of 100000 yuan. Before the turning point are small fires that have an obvious stable power-law relation of the frequency-loss distribution, while after the turning points are major fires for which we cannot obtain an exact power-law relation owing to the insufficiency of data, especially those major fires with direct economic losses exceeding millions of yuan or even up to tens of millions of yuan, which rarely occur in a city like Hefei. The occurrence probability of major fires is calculated employing the established prediction model of extreme values. We choose the greatest loss of the order of 100000 yuan among the annual fire loss data for 2000 to 2006 (shown in Table 1) and put the value into eq. (7) to generate seven linear equations for $a$ and $b$, and then solve the equations employing the OLS method.

The calculated values of $a$ and $b$ are $a=0.3$ and $b=1.18$. We assume that the maximum loss $L_{\max }$ is 1 million yuan and then 10 million yuan; then according to eq. (8), the corresponding occurrence probability of major fires can be calculated, as shown in Table 2.

It is seen that within the next five years, the occurrence probability of a major fire with direct economic loss of more than 1 million yuan is 0.83 , while that for a major fire with direct economic loss of more than 10 million yuan is 0.59 . With extension of the time period for prediction, the corresponding values of probability increase. This indicates that the occurrence probability of major fires will continue to increase over a long period. As reported in the [16], there is a certain correlation between the situation of urban fire and the economy in that the economic loss and/or frequency of urban fire has a rising trend in the earlier stage of economic take-off. As a developing city, Hefei is now in a stage of rapid economic growth, as shown in Figure 5; thus, our results are also accordant with the relation between the economy and urban fire. Statistics show that in 2007, the largest direct economic loss caused by urban fire in Hefei exceeded 4 million yuan, that in 2008 exceeded 6 million yuan, and in 2009, a major fire in the Zhougudui wholesale market for agricultural products resulted in a direct

Table 1 Largest loss of the order of 100000 yuan from 2000 to 2006 in the city of Hefei

\begin{tabular}{cc}
\hline Year & $L_{\max }(10000$ yuan $)$ \\
\hline 2000 & 10.6676 \\
2001 & 12.0000 \\
2002 & 19.0462 \\
2003 & 14.4645 \\
2004 & 11.7273 \\
2005 & 14.9391 \\
2006 & 10.6544 \\
\hline
\end{tabular}

Table 2 Occurrence probability of fire incidents with the logarithm of direct economic loss not less than $M\left(M=\ln L_{\max }\right)$

\begin{tabular}{ccc}
\hline$T$ (Year $)$ & $\begin{array}{c}\text { Occurrence probability } \\
P\left(L_{\max }=100000 \text { yuan }\right)\end{array}$ & $\begin{array}{c}\text { Occurrence probability } \\
P\left(L_{\max }=100000 \text { yuan }\right)\end{array}$ \\
\hline 5 & 0.83 & 0.59 \\
10 & 0.97 & 0.83 \\
15 & 1 & 0.93 \\
\hline
\end{tabular}

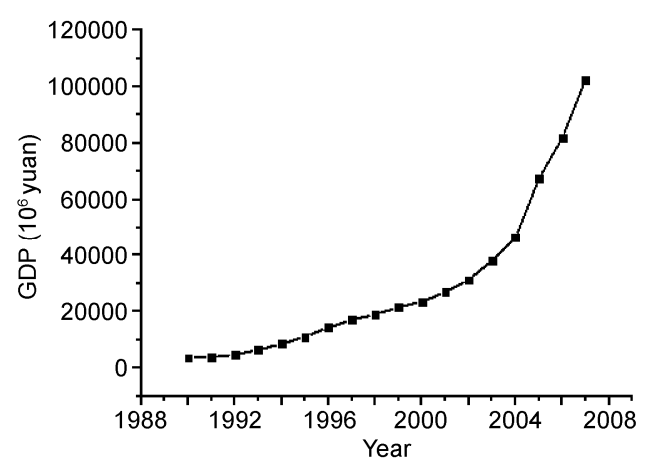

Figure 5 Increasing trend of the gross domestic product in Hefei. 
economic loss exceeding 10 million yuan. It is seen from these statistics that the results of major fire prediction are basically accordant with the real major fire situation in $\mathrm{He}$ fei. Moreover, with the extension of the time period for prediction, the results become more accordant with the real situation. However, when the time period for prediction exceeds 15 years, the occurrence probabilities of there being major fires resulting in economic losses on the orders of 1 million yuan and 10 million yuan both reach $100 \%$, which indicates that this model will be useful in providing quantitative results for predicting the major fire situation of Hefei in a proper time period such as 15 years and providing scientific suggestions to the fire department to deal with the major fire risk.

\section{Conclusions}

In this paper, we analyzed the spatial (economic loss) and temporal characteristics of urban fires employing relevant statistical methods. It was found that the urban fire system has a fractal structure on a spatial scale in terms of a power-law relation between fire frequency and economic loss, whereas there is no temporal fractal structure (i.e., no $1 / f$ noise); there is a stable exponential distribution on the temporal scale instead of a power-law distribution. This indicates that the urban fire system does not meet the rigorous criteria of SOC. Subsequently, a correlation model of the fire frequency and economic loss is established employing the extremum statistical method, which proves that the limit of the extreme value distribution of direct economic loss caused by urban fire converges to the Gumbel (I) formula. Using this model, the specific fire situation of $\mathrm{He}$ fei is analyzed as a case study and the results are found to be accordant with the relation between the economy and urban fires. The relation, to a certain extent, can be used to make scientific and reasonable predictions of major fires for which there is relatively insufficient statistical data based on small fires with sufficient data, especially for small or medium cities. We will further focus on factors that may affect the stability of prediction results. The urban fire system is a complex system subjected to the coupled effects of socio- economic factors, the environment, and human activities. Thus, if we can relate these factors to the statistical laws of urban fires in terms of introducing calibration parameters into the prediction algorithm for a specific city and making real-time correction of the prediction state, the outcomes will be much more useful for the prevention of urban fires.

This work was supported by the National Natural Science Foundation of China (91024025).

1 Bak P, Tang C, Wiesenfeld K. Self-organized criticality: An explanation of 1/f noise. Phys Rev Lett, 1987, 59: 381-384

2 Hwa T, Kardar M. Fractals and self-organized criticality in dissipative dynamics. Phys D, 1989, 38: 198-202

$3 \mathrm{Yu}$ Q, Guo J B. Statistics and self-organized criticality characters of blackouts in China electric power systems. Aut Elec Pow Sys, 2006, 30: 16-21

4 Yao Y, Yao L K. Applicability of self-organized criticality in epidemic spreading. J Catastrophol, 2008, 23: 20-23

5 Gao Zh N, Yao L K, Yang Q H, et al. Study on the relationship between active faults and seismicity based on the SOC theory. J Seismol Res, 2009, 32: 119-124

6 Drossel B, Schwabl F. Self-organized critical forest-fire model. Phys Rev Lett, 1992, 69: 1629-1632

7 Song W G, Wang B H, Shu L F. Self-organized criticality and macro-characteristics of forest fire system. J Grad Sch Chin Acad Sci, 2003, 20: 205-211

8 Song W G, Wang Q, Wang J. Characteristics of fire distribution in Huludao City and its enlightenment to city fire danger analysis. Sci Technol Rev, 2005, 23: 35-37

9 Akkaya D A, Yücemen M S. Estimation of earthquake hazard based on extremes of local integral random functions. Eng Geol, 2000, 58: 53-66

10 Katz R W, Parlange M B, Naveau P. Statistics of extremes in hydrology. Adv Wat Res, 2002, 25: 1287-1304

11 Dirk N R. Extreme value theory of generalized order statistics. J Stat Plan Infer, 1996, 55: 281-297

12 Majumdar S N, Krapivsky P L. Extreme value statistics and traveling fronts: Various applications. Phys A, 2003, 318: 161-170

13 Jing G X, Wang W M, Hao T X. Fire accident forecast based on gray-back propagation networks. Ind Saf Env Prot, 2008, 34: 61-63

14 Zhou A T, Jing G X, Sun G, et al. Application of improved grey model in fire accident forecasting. J Saf Sci Technol, 2006, 2: 62-64

15 Jing G X, Wang W M, Liu Q J. Application of information diffusion theory in risk assessment of fire in Henan province. Ind Saf Env Prot, 2008, 34: 62-64

16 Yang L Z, Jiang D B. The relationship between fire and socioeconomic factors in China. Eng Sci, 2003, 5: 62-67

Open Access This article is distributed under the terms of the Creative Commons Attribution License which permits any use, distribution, and reproduction in any medium, provided the original author(s) and source are credited. 